MATHEMATICS OF COMPUTATION

Volume 67, Number 223, July 1998, Pages 1163-1172

S 0025-5718(98)00950-8

\title{
FAST EVALUATION OF MULTIPLE ZETA SUMS
}

\author{
RICHARD E. CRANDALL
}

ABStract. We show that the multiple zeta sum:

$$
\zeta\left(s_{1}, s_{2}, \ldots, s_{d}\right)=\sum_{n_{1}>n_{2}>\ldots>n_{d}} \frac{1}{n_{1}^{s_{1}} n_{2}^{s_{2}} \ldots n_{d}^{s_{d}}},
$$

for positive integers $s_{i}$ with $s_{1}>1$, can always be written as a finite sum of products of rapidly convergent series. Perhaps surprisingly, one may develop fast summation algorithms of such efficiency that the overall complexity can be brought down essentially to that of one-dimensional summation. In particular, for any dimension $d$ one may resolve $D$ good digits of $\zeta$ in $O(D \log D / \log \log D)$ arithmetic operations, with the implied big- $O$ constant depending only on the set $\left\{s_{1}, \ldots, s_{d}\right\}$.

\section{INTRODUCTION}

The multiple zeta sums:

$$
\zeta\left(s_{1}, s_{2}, \ldots, s_{d}\right)=\sum_{n_{1}>n_{2}>\ldots>n_{d}} \frac{1}{n_{1}^{s_{1}} n_{2}^{s_{2}} \ldots n_{d}^{s_{d}}},
$$

also called Euler/Zagier sums, have attracted considerable interest in recent times (see Bailey et al. [3], Borwein et al. [4], Borwein et al. [7], Broadhurst et al. [9], Crandall and Buhler [11], Markett [13] and Zagier [14]) (note that some previous treatments have reversed ordering of the indices $n_{i}$ ). The study of such sums is not only important to general zeta function theory, but also touches upon such domains as knot theory and particle physics methodology. To simplify our present analysis we shall concentrate on this scenario: each $s_{i}$ is a positive integer and, to ensure convergence of the explicit sum, $s_{1}>1$. It should be noted, however, that even in absence of the integer restriction on the $s_{i}$, the ideas herein do lead to the development of convergent, albeit more intricate series.

These multiple zeta sums have been given many attractive, exact evaluations. It could be said that the research situation is quite rich, in the following sense. Whereas Euler's original evaluations, of which

$$
\zeta(4,1)=2 \zeta(5)-\zeta(2) \zeta(3)
$$

is exemplary, along with more modern evaluations such as:

$\zeta(4,5,1)=2 \zeta(7,3)+\zeta(5)^{2}-17 \zeta(3) \zeta(7)+\zeta(2) \zeta(5,3)+10 \zeta(2) \zeta(3) \zeta(5)-\frac{21}{20} \zeta(10)$,

Received by the editor September 30, 1996 and, in revised form, March 3, 1997.

1991 Mathematics Subject Classification. Primary 11Y60, 11Y65; Secondary 11M99.

(C)1998 American Mathematical Society 


$$
\zeta(6,6,6,6)=\frac{4 \pi^{24}}{432684797065192546875},
$$

are now proved (Markett [13], Borwein and Girgensohn [8], Borwein et al. [7]), suspected evaluations such as Zagier's conjectured:

$$
\zeta(3,1,3,1, \ldots, 3,1)=\frac{2 \pi^{4 n}}{(4 n+2) !}
$$

(where $n$ denotes the the number of $(3,1)$ pairs) remain elusive.* Even the twodimensional sums $\zeta(6,2)$ and $\zeta(3,5)$ are unknown, in the sense that neither has been cast in a finite form involving "one-dimensional" sums such as Riemann zeta values. There is a growing literature on which sums are evaluable, which can be reduced to lower-dimensional forms, and which appear dimensionally irreducible and therefore "fundamental." For example, in Borwein and Girgensohn [8] it is argued that 3-dimensional sums can always be reduced (to lower-dimensional forms) if $s_{1}+s_{2}+s_{3}$ is either even or less than 10 . On the other hand, $\zeta(5,3,3)$ has never been expressed as a combination of, say, two-dimensional $\zeta$ sums, "one-dimensional" Riemann sums, and fundamental constants.

To verify conjectured evaluations, indeed to generate reasonable conjectures, it has been important to be able to evaluate $\zeta$ numerically, to high precision (say $>100$ decimal digits). The requirement of high precision arises for various reasons, such as the desire to avoid "accidental" relations and to guard against mishap when some integral coefficients of exact relations are large. The original approach of Bailey et al. [3] involved an Euler-Maclaurin scheme for estimating double sums. This approach has been effectively generalized by Broadhurst, as described in [7], to higher dimensions. A primary difficulty with an Euler-Maclaurin approach is that explicitly convergent sums do not obtain. In the special case of double sums, Crandall and Buhler [11] developed convergent series for $\zeta(r, s)$, where $r, s$ need not even be integers. In this way, both $\zeta(6,2)$ and $\zeta(3,5)$ are now numerically resolved, each to more than 1200-digit precision (Bailey [1]). Given the present complexity claim, it should be possible to attain such extreme precision for sums of much higher dimension.

For the $d$-dimensional, unified approach we consider herein, the resulting convergent series will involve a single free parameter, $\lambda$, restricted to the interval $[0,2 \pi)$. One advantage of such free-parameter expansions is that one may gain substantial confidence in the numerical scheme by repeating the convergent sum for different $\lambda$ choices and verifying that the numerical result is, to some satisfactory precision, invariant.

\section{INTEGRAL REPRESENTATION}

Our series development starts with the observation that $\zeta$ admits of certain integral representations (see Crandall [10], Borwein et al. [7], and Zagier [14]). Denote the argument vector $\vec{s}=\left\{s_{1}, s_{2}, \ldots, s_{d}\right\}$, and consider a particular $d$-dimensional integral representation:

$$
\zeta(\vec{s})=\sum_{m_{h} \in Z^{+}} \int_{0}^{\infty} \cdots \int_{0}^{\infty} \prod_{i} x_{i}^{s_{i}-1} e^{-x_{i} \sum_{h=i}^{d} m_{h}} \frac{d x_{i}}{\Gamma\left(s_{i}\right)}
$$

\footnotetext{
* Note added in proof: D. Broadhurst has recently announced a proof of this conjecture of Zagier.
} 
in which indices $i, h$ are understood each to run through values $1, \ldots, d$. This representation is proved quickly by performing the $d$ individual (and separated) integrals, then re-indexing, with the $n_{i}$ in (1.1) becoming appropriate partial sums of the $m_{h}$.

An immediate transformation of variables yields a more practical representation. Set

$$
u_{k}=\sum_{i=1}^{k} x_{i}
$$

for $k=0,1, \ldots, d$, and interpret $u_{0}=0$ for convenience in what follows. Now the Jacobian of the $\vec{x} \rightarrow \vec{u}$ transformation is conveniently the identity, and we have

$$
\zeta(\vec{s})=\sum_{m_{h} \in Z^{+}} \int_{u_{d}>u_{d-1}>\ldots>u_{0}} \prod_{i}\left(u_{i}-u_{i-1}\right)^{s_{i}-1} e^{-u_{i} m_{i}} \frac{d u_{i}}{\Gamma\left(s_{i}\right)},
$$

where, again, each of $i, h$ runs through $1, \ldots, d$. The integrand is now somewhat more palatable, but at the expense of a more complicated domain of integration. This domain can be partitioned in the following way. Choose a positive parameter $\lambda$, and consider subdomains of integration defined for each of $k=0,1, \ldots, d$ as:

$$
u_{d}>\ldots>u_{k+1}>\lambda>u_{k}>\ldots>u_{0},
$$

where for $k=d$ the inequality chain to the left of the $\lambda$ is interpreted as empty. It is evident that the domain of integration in (2.3) can be taken to be the disjoint union of these $(d+1)$ subdomains. Now for the subdomain indexed by a $k<d$ we can expand the term $\left(u_{k+1}-u_{k}\right)^{s_{k+1}-1}$ in a finite binomial series, and in this way obtain a finite set of once-separated integrals. (This expansion step is, in fact, the only juncture at which the integer restriction on $s_{k}$ is critical.) For the "lower" part of an integral, that is over variables $u_{1}, \ldots, u_{k}$, we sum formally over $m_{1}, \ldots, m_{k}$. In this way we obtain a kind of "factored" representation into lower and upper integrals:

$$
\begin{aligned}
\zeta(\vec{s})= & Y(\vec{s} ; 0 ; \lambda)+Z(\vec{s} ; \lambda) \\
& +\sum_{k=1}^{d-1} \sum_{q=0}^{s_{k+1}-1} \frac{(-1)^{q}}{q !} Y\left(s_{1}, \ldots, s_{k} ; q ; \lambda\right) Z\left(s_{k+1}-q, s_{k+2}, \ldots, s_{d} ; \lambda\right),
\end{aligned}
$$

where the "lower" integrals $Y$ are defined:

$$
Y\left(s_{1}, \ldots, s_{k} ; q ; \lambda\right)=\int_{\lambda>u_{k}>\ldots>u_{1}>0} u_{k}^{q} \prod_{i=1}^{k} \frac{\left(u_{i}-u_{i-1}\right)^{s_{i}-1}}{e^{u_{i}}-1} \frac{d u_{i}}{\Gamma\left(s_{i}\right)},
$$

while the "upper" integrals are

$$
Z\left(r_{1}, \ldots, r_{j} ; \lambda\right)=\sum_{m_{h} \in Z^{+}} \int_{u_{j}>u_{j-1}>\ldots>u_{1}>\lambda} \prod_{i=1}^{j}\left(u_{i}-u_{i-1}\right)^{r_{i}-1} e^{-u_{i} m_{i}} \frac{d u_{i}}{\Gamma\left(r_{i}\right)} .
$$

Note that in (2.4), when $k=d-1$ the argument vector for $Z$ is interpreted to be the singleton term $s_{d}-q$; i.e., the subsequence $s_{k+2}, \ldots, s_{d}$ is empty. Note also that evaluation of the Riemann $\zeta$ involves only the first two terms, a $Y$ and a $Z$, in (2.4).

The relation (2.4) shows that, for any positive $\lambda$, every multiple zeta can be written as a finite sum of products of $Y, Z$ functions. In the next section we show that, by way of proper constraint on the parameter $\lambda$, each of the necessary $Y$ and $Z$ may be cast in efficient convergent series form, whence a general multiple zeta can be calculated efficiently from (2.4). 


\section{Operator CAlculus AND Bernoulli numbers}

For all $r_{1}, \ldots, r_{j}$ positive integers we can rewrite the general $Z$ integral (2.6) by replacing terms $\left(u_{i}-u_{i-1}\right)^{r_{i}-1}$ with respective operators

$$
\left(-\partial_{m_{i}}+\partial_{m_{i-1}}\right)^{r_{i}-1}
$$

then moving these operators out of the integral. We then re-index the $m_{i}$ indices according to

$$
n_{i}=\sum_{h=i}^{d} m_{h}
$$

whence the transformed derivative operators $\partial_{n_{i}}$ collapse conveniently to yield

$$
Z\left(r_{1}, \ldots, r_{j} ; \lambda\right)=\frac{1}{\Gamma\left(r_{1}\right)} \sum_{n_{1}>n_{2}>\ldots>n_{j}}\left(-\partial_{n_{1}}\right)^{r_{1}-1} \frac{e^{-\lambda n_{1}}}{n_{1}} \frac{1}{n_{2}^{r_{2}} \ldots n_{j}^{r_{j}}},
$$

where symbolic differentiation is understood to be performed prior to the summation. It is evident that $Z$ looks very much like the original multiple zeta definition. Indeed $Z$ coincides with $\zeta$ as $\lambda \rightarrow 0$, a fact also formally evident from (2.4) because said $\lambda$ limit pinches all $Y$ integrals to zero.

Now (3.1) is a patently convergent series, more efficient for larger $\lambda$. It remains to develop a converging series for the lower $Y$ integrals. For $\lambda<2 \pi$ we can expand each term $\left(e^{u_{i}}-1\right)^{-1}$ within the definition (2.5), in standard Bernoulli series. Such expansion leads to

$$
Y\left(s_{1}, \ldots, s_{k} ; q ; \lambda\right)=\sum_{n_{i} \geq 0} \prod_{p=1}^{k} \frac{B_{n_{p}}}{n_{p} !} \int_{u_{k}>u_{k-1}>\ldots>u_{0}} u_{k}^{q} \prod_{i=1}^{k}\left(u_{i}-u_{i-1}\right)^{s_{i}-1} \frac{d u_{i}}{\Gamma\left(s_{i}\right)} .
$$

This time we avoid expanding the polynomials, using instead a change of variables $u_{i}=v_{i} u_{i+1}$, with $v_{0}:=0$ and, for convenience, $u:=u_{k}$, to yield

$$
\begin{aligned}
& Y\left(s_{1}, \ldots, s_{k} ; q ; \lambda\right) \\
& \quad=\sum_{n_{i} \geq 0} \prod_{p=1}^{k} \frac{B_{n_{p}}}{n_{p} !} \int_{0}^{\lambda} u^{S+N+q-k-1} d u \prod_{i=1}^{k} \int_{0}^{1} v_{i}^{S_{i}+N_{i}-i-1}\left(1-v_{i-1}\right)^{s_{i}-1} \frac{d v_{i}}{\Gamma\left(s_{i}\right)},
\end{aligned}
$$

where we have introduced partial sum nomenclature:

$$
N_{p}=n_{1}+\ldots+n_{p}, \quad S_{p}=s_{1}+\ldots+s_{p}
$$

with $N:=N_{k}, S:=S_{k}$. Each $v$-integral is a beta function expressible in terms of gamma functions, so we immediately obtain the final result, an explicit series for $Y$,

$$
\begin{aligned}
& Y\left(s_{1}, \ldots, s_{k} ; q ; \lambda\right) \\
& \quad=\frac{1}{\Gamma\left(s_{1}\right)} \sum_{n_{i} \geq 0} \frac{\lambda^{N+S+q-k}}{N+S+q-k} \prod_{p=1}^{k} \frac{B_{n_{p}}}{n_{p} !} \prod_{m=1}^{k-1} \frac{\Gamma\left(N_{m}+S_{m}-m\right)}{\Gamma\left(N_{m}+S_{m+1}-m\right)},
\end{aligned}
$$

where the indices $n_{1}, \ldots, n_{k}$ are restricted to non-negative integers. It turns out that the sum (3.2) for $Y$ always converges absolutely when $\lambda<2 \pi$.

The relations $(2.4),(3.1),(3.2)$ comprise a complete prescription for computation of multiple zeta values. It turns out that considerable reduction in the apparent complexity of the $Y, Z$ sums is possible, as we see in the next section. 


\section{Summation collapse and Bailey acceleration}

On the face of it, the $Z$ sum (3.1) seems to require, for first index $n_{1}$ summed over the range $j \leq n_{1}<L_{1}$, at least $O\left(L_{1}^{j}\right)$ operations; for after all, the sum is over a $j$-dimensional lattice region. The same basic dimension-dependent complexity would also seem to obtain for the $Y$ sum, (3.2). However, both of these preliminary estimates turn out to be overwhelmingly pessimistic, as we presently explain.

It turns out that the $Z$ sum can be thought of, in a computational sense to be made precise later, as one-dimensional. Speaking heuristically, whenever the first index $n_{1}$ in (3.1) increments, not much new information is contributed from the other indices. Specifically, the sum, which might originally be implemented as a $j$ nested loop, can be collapsed into just one loop. Here is a pseudocode construction for the collapsed $Z$ sum. Denote the operator part of (3.1) by

$$
f(n, r, \lambda):=\left(-\partial_{n}\right)^{r-1} \frac{e^{-\lambda n}}{n} .
$$

Then the summation collapse algorithm starts with initialization of $(j+1)$ variables and proceeds with a singly-nested loop, like so:

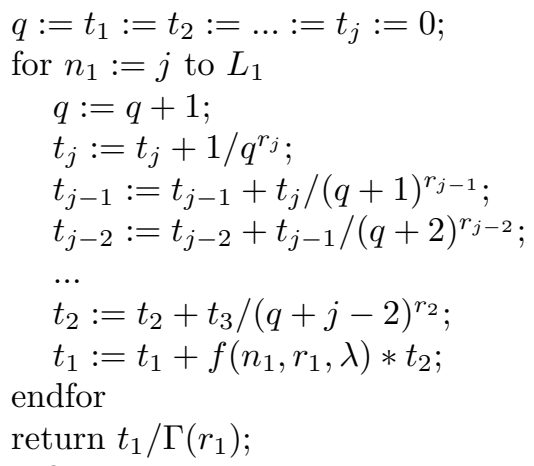

The final returned value, which approximates $Z$ to order $e^{-\lambda L_{1}}$, is obtained thus in asymptotically $j L_{1}$ loop steps, or $O\left(L_{1}\right)$ arithmetic operations. It is in this precise sense that we may think of the $Z$ sum's complexity as essentially that of a one-dimensional sum.

In a practical implementation it is clearly advantageous to pre-compute all needed values of $f(n, r, \lambda)$ in an initialization phase. To this end it should be pointed out that the multiterm expression resulting from the differentiation indicated in the definition of $f(n, r, \lambda)$ can be generated with a simple recursion. Also, for a fixed $r$ and $\lambda$, the expression for $f$ satisfies a recursion in $n$, so that repeated evaluations of the exponential function are not required. Thus an economical procedure to compute the required $f$ values can be stated as follows. Let $r_{m}$ denote largest $r_{k}$ that will be encountered anywhere in the full computation, i.e. the maximum of the original $s$ vector. Then perform the following algorithm:

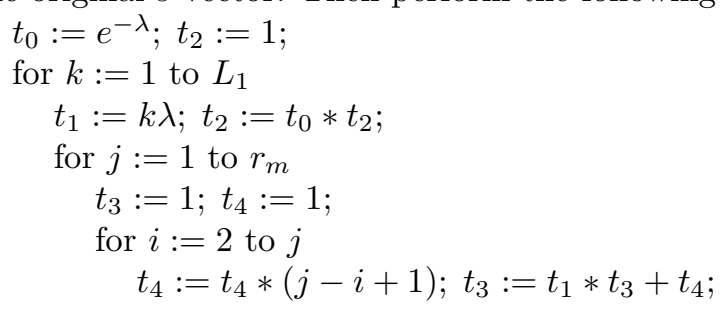




$$
\begin{aligned}
& \text { endfor } \\
& \qquad f(k, j, \lambda):=t_{2} * t_{3} * k^{-j} ; \\
& \text { endfor } \\
& \text { endfor }
\end{aligned}
$$

The collapse of $Z$ sums leaves only the problematic $Y$ sums which do not, unfortunately, enjoy an analogous collapse. But all is not lost, thanks to an acceleration first observed by Bailey [1]. He noticed that certain two-dimensional series implementations in [11] could benefit from convolution algorithms. Let us look, as a canonical example, at the problem of evaluating $\zeta(6,2)$. In (2.4), and given knowledge of $Z$ sum collapse, there remains only one problematic two-dimensional sum, which can be written in the following suggestive style:

$$
Y(6,2 ; 0 ; \lambda)=\frac{1}{\Gamma(6)} \sum_{N_{2} \geq 0} \frac{\lambda^{N_{2}+6}}{N_{2}+6} \sum_{0 \leq N_{1} \leq N_{2}} \frac{B_{N_{1}} B_{N_{2}}-N_{1}}{N_{1} !\left(N_{2}-N_{1}\right) !} \frac{1}{\left(N_{1}+5\right)\left(N_{1}+6\right)}
$$

We expect to perform the outer summation over a constrained range: $0 \leq N_{2} \leq L_{2}$, to obtain an error of order $\left(\frac{\lambda}{2 \pi}\right)^{L_{2}}$. We observe that the inner sum in (4.1) is in fact the $N_{2}$-th element of an acyclic convolution. The convolution procedure is: first, find the acyclic convolution (equivalent to straightforward polynomial multiplication) of the two sequences (of coefficients):

$$
\begin{gathered}
x_{1}:=\left\{\frac{B_{n}}{n !(n+5)(n+6)} ; n=0,1,2, \ldots, L_{2}\right\}, \\
x_{2}:=\left\{\frac{B_{n}}{n !} ; n=0,1,2, \ldots, L_{2}\right\} .
\end{gathered}
$$

Then the $Y$ value in (4.1) can be obtained simply from the elements of the acyclic convolution of $x_{1}, x_{2}$ (which elements are also the coefficients of the relevant product polynomial).

In a practical implementation it is important to efficiently compute the Bernoulli terms $B_{0} / 0$ ! through $B_{L_{2}} / L_{2}$ !, even if this be done only once in a global initialization phase. Fortunately, it turns out that these Bernoulli terms can be obtained in a "parallel" fashion via a method pioneered by J. P. Buhler; namely, Newton method inversion applied to the formal generating function

$$
\frac{z}{e^{z}-1}=\sum_{k=0}^{\infty} \frac{B_{k}}{k !} z^{k}
$$

One uses the series expansion of $\left(e^{z}-1\right)$ through a desired degree $L_{2}$ in $z$, then inverts the resulting polynomial, the procedure requiring $O\left(L_{2} \log L_{2}\right)$ arithmetic operations ([10], [2]). Since the convolution inherent in (4.1) can be performed, via FFT methods, say, also in $O\left(L_{2} \log L_{2}\right)$ arithmetic operations, it follows from all the considerations above on $Y, Z$ that $\zeta(6,2)$ can be obtained to $D$ good decimal digits in $O\left(L_{1}\right)+O\left(L_{2} \log L_{2}\right)$ arithmetic operations, where each of the outer summation limits $L_{1}, L_{2}$ has been chosen such that the respective sums are both good to the $D$ digits.

Happily, for dimensions $d>2$, the $Y$ sum is still susceptible to a convolution approach. Write the double product in (3.2) abstractly, as

$$
\frac{B_{N_{1}}}{N_{1} !} \frac{B_{N_{2}-N_{1}}}{\left(N_{2}-N_{1}\right) !} \ldots \frac{B_{N_{k}-N_{k-1}}}{\left(N_{k}-N_{k-1}\right) !} g_{1}\left(N_{1}\right) \ldots g_{k-1}\left(N_{k-1}\right) .
$$


Now observe that the sum over $N_{1}$ in the range $0 \leq N_{1} \leq N_{2}$, which sum involving only the first two Bernoulli terms and the $g_{1}$ term, is but a certain convolution evaluated at the $N_{2}$-th element, as before. But this means that the sum over $0 \leq N_{2} \leq N_{3}$ can then be performed via convolution of one sequence indexed by $N_{2}$ and involving $g_{2}$, with another sequence indexed by $N_{3}-N_{2}$, and so on.

A pseudocode realization of the entire convolution procedure can be formulated as follows. Choose the outer summation limit $L_{2}$ to be one less than a power of 2 (a convenience, so that convolution lengths are themselves powers of 2). Now define sequences, each of length $L_{2}+1$, as follows:

$$
B:=\left\{\frac{B_{n}}{n !} ; n=0,1,2, \ldots, L_{2}\right\},
$$

and for $m=1, \ldots, k-1$,

$$
G_{m}:=\left\{\frac{\Gamma\left(n+S_{m}-m\right)}{\Gamma\left(n+S_{m+1}-m\right)} ; n=0,1,2, \ldots, L_{2}\right\} .
$$

Define further, for two sequences $X, Y$ each of length $L$, their "half-cyclic" convolution as the first $L$ elements of the full acyclic convolution, and denote this half-cyclic by $X \times_{H} Y$. This half-cyclic can be obtained via these steps: zero-pad each of $X, Y$ to length twice $L$, perform the cyclic convolution of the padded sequences (via standard FFT methods, say), and finally, take the first $L$ elements of the result. Further, we denote by $X * Y$ the dyadic (elementwise) product sequence of any two equal-length sequences $X, Y$. Now initialize a sequence $X$ and carry out a loop, as follows:

$$
\begin{aligned}
& X:=B ; \\
& \text { for } m:=1 \text { to } k-1 \\
& X:=X * G_{m} ; \\
& X:=X \times_{H} B ; \\
& \text { endfor }
\end{aligned}
$$

Now the $Y$ value from the defining series (3.2) is obtained simply as a weighted sum over elements $X[N]$ of the final half-cyclic, as

$$
Y=\frac{1}{\Gamma\left(s_{1}\right)} \sum_{N=0}^{L_{2}} \frac{\lambda^{N+S+q-k}}{N+S+q-k} X[N] .
$$

The convolution procedure admits of interesting potential enhancements. First, if FFT method is used, the transform of the (zero-padded) $B$ sequence may be done just once, and used repeatedly for the half-cyclic within the loop. Second, since every odd-indexed Bernoulli number vanishes except $B_{1}=-1 / 2$, the $B$ sequence is sparse. One may, in principle, algebraically subtract out all dependence on $B_{1}$ from (3.2), whence convolutions lengths may be effectively halved. Third, halfcyclic convolution can be done via cyclic and negacyclic convolution each of half length (sans zero-padding), and even done in a special, recursive fashion for lengths not a power of 2 [10]. Perhaps the most intriguing possible enhancement is to somehow invoke the negacyclic option to effect the whole pseudocode loop via one long dyadic product of various initial transforms. In other words, there may exist some means to generate " $(k-1)$-fold half-cyclic convolutions" in such a maximally efficient manner. 
We have thus realized the considerable advantage of the Bailey acceleration: if proper precision is maintained for each convolution, we see that the total operation cost is still that of the $(k=1)$-dimensional case; namely $O\left(L_{2} \log L_{2}\right)$.

\section{Complexity}

It follows from all the above complexity estimates that, if the respective outer summation limits $L_{1}, L_{2}$ be equal, then the multiple zeta with pure-integer argument vector $\vec{s}$ can be resolved to $D$ digits in $O(D \log D)$ arithmetic operations. But we can optimize a little further. Since the complexity for the $Z$ sum is slightly lower (i.e. linear in $L_{1}$ ), the optimal parameter $\lambda$ can be slightly lowered (with $\left.L_{1} \sim L_{2} \log D\right)$ to yield an overall complexity of $O(D \log D / \log \log D)$. The problem of what should be the overall big- $O$ constant is interesting. Perhaps it is reasonable to conjecture that the constant can be taken to be some simple function only of the dimension $d$ and the "weight" $\sum_{i=1}^{d} s_{i}$. (In this regard, note Bailey's empirical discovery of quadratic growth (in dimension $d$ ) of this constant for a particular class of zetas, as we discuss in the next section.) Incidentally, many existing schemes for evaluation of, say, an arbitrary Riemann $\zeta(n), n \in Z$, require $O(D \log D)$ operations or slightly better than this [10]; although there are elegant, Apery-like formulae involving $O(D)$ operations and small implied big- $O$ constants [6]; and a complicated but asymptotically efficient scheme involving only $O\left(M(D) \log ^{2} D\right)$ bit operations [12], where $M(D)$ is the bit-complexity of multipication of two $D$-digit numbers. It may also be possible, for our present multiple zeta algorithms or the cited Riemann zeta ones, to effect a hypergeometric acceleration, as in [5] for further reduction in operation complexity, to say $O\left(D^{a}\right)$ for some $a<1$. In any event it is clear that the expedient of summation collapse combined with Bailey acceleration is efficient indeed: our $d$-dimensional sums reduce, up to a big- $O$ constant, to our own $(d=1)$-dimensional case. If an implementation be sufficiently adroit, the penalty for higher dimensions should be correspondingly light.

\section{EXPERIMENT AND PRACTICAL CONSIDERATIONS}

Even if the acceleration of section four is implemented, the computation of the $Y$ function is generally more expensive than the $Z$ function. If the acceleration is not employed, the disparity between these two costs is even more pronounced. Fortunately, these costs can be brought more into balance, with an overall savings in computation time, by adjusting the value of $\lambda$. Smaller values of $\lambda$ tend to lower the cost of the $Y$ function, but increase the cost of the $Z$ function. To be precise, the two limits $L_{1}$ (the limit for the $Z$ function) and $L_{2}$ (the limit for the $Y$ function) are given by

$$
L_{1}=\frac{-\log \epsilon}{\lambda}, \quad L_{2}=\frac{\log \epsilon}{\log (\lambda /(2 \pi))},
$$

where $\epsilon$ is the desired accuracy of the result. Using these formulae, an optimal value of $\lambda$ can be determined.

The author employed this asymmetrical technique to evaluate the fourdimensional sum $\zeta(3,1,3,1)$, using $\lambda=1 / 20, L_{1}=12000$ and $L_{2}=120$, so that the collapsed loop for $Z$ is about as fast as the straightforward (i.e. without acceleration) evaluation of $Y$ using (3.2). The numerical result agrees with Zagier's conjectured $\zeta(3,1,3,1)=2 \pi^{8} / 10$ ! to more than 250 decimal digits. 
At the time of this writing, the present author has been informed by David H. Bailey that the latter has succeeded implementing the complete procedure described herein, including the convolution acceleration for the $Y$ function. Bailey employed $\lambda=5 / 32=0.15625, L_{1}=12000$ and $L_{2}=512$, which yields more than 800 decimal digit accuracy in the result. The resulting program verified $\zeta(3,1,3,1)=2 \pi^{8} / 10$ !, to 800 decimal place accuracy, in only four minutes run time on an IBM RS6000/590 workstation.

Bailey has further verified the Zagier conjecture, as it is stated in our inroduction, for $1 \leq n \leq 10$, again to 800 decimal place accuracy for each number $n$ of argument pairs $\{3,1\}$. The entire run over the stated range of $n$ required just 4.3 hours on an IBM RS6000/590 workstation. Bailey found that the CPU time required for each instance $n$ (not including global initialization, such as computing the Bernoulli coefficients used for all dimensions), accurately satisfies an empirical quadratic formula in $n$. These results suggest that evaluations of multiple zetas of very high dimensions (perhaps dimensions into the hundreds) can be feasibly computed using the techniques described herein.

There are many useful, sharp tests of any numerical algorithm for mutiple zetas. The compendium [7] contains many fascinating exact evaluations, as well as various well-motivated conjectures. One way to test higher-dimensional implementations is to exploit the complete reducibility of any multiple zeta of the form $\zeta(s, s, \ldots, s)$; i.e. with all argument components equal. Denote such a $\zeta$ having exactly $N$ appearances of $s$ by $\zeta\left(\{s\}_{N}\right)$. Then, via a combinatorical argument, one may establish the recursion

$$
\zeta\left(\{s\}_{N}\right)=\frac{1}{N} \sum_{j=1}^{N}(-1)^{j-1} \zeta(j s) \zeta\left(\{s\}_{N-j}\right),
$$

where we also define the empty case $\zeta(\phi):=1$, revealing immediately that any $\zeta\left(\{s\}_{N}\right)$ can always be reduced to "one-dimensional" (Riemann zeta) sums. The exact evaluation of $\zeta(6,6,6,6)$ exhibited in the Introduction may be effected in this way. Another rigorous test for any implementation is the sum rule [7]:

$$
\sum_{n_{j}>\delta_{j, 1} ; \sum n_{j}=N} \zeta\left(n_{1}, \ldots, n_{d}\right)=\zeta(N) .
$$

Finally, we address briefly the scenario in which at least one of the argument components is not an integer. It turns out that the $Z$ integral defined by (2.6) can always be cast as a sum of the type (3.1), except that for $r_{1}$ non-integral we use the correspondence

$$
\left(-\partial_{n_{1}}\right)^{r_{1}-1} \frac{e^{-\lambda n_{1}}}{n_{1}} \leftrightarrow \frac{\Gamma\left(r_{1}, \lambda n_{1}\right)}{n_{1}^{r_{1}}},
$$

where $\Gamma$ here is the incomplete gamma function. The $Y$ sum (3.2) is, as it stands, a correct representation of the defining form (2.5) for any set of real $s_{1}, \ldots, s_{k}$, as long as the sum converges. Therefore, the only thing preventing a complete series development for arbitrary real argument components is that the factored representation (2.4) involves explicit summation indices $s_{k+1}-1$ which must be integers. Thus more work must be put into the expansion of the integrand of (2.3) in order to handle arbitrary real argument vector components. However, as $s_{1}$ in particular does not appear as a summation index, we can certainly evaluate, via 
the tools already in hand, such a priori poorly convergent entities as

$$
\begin{aligned}
& \zeta\left(\frac{3}{2}, 1\right) \\
& =4.68181441155622703569221027933719959407392140996102081308586936 \ldots, \\
& \quad \zeta\left(\frac{3}{2}, 1,1\right) \\
& \quad=9.124484559118879556428450796505756958335362902190857985302503 \ldots \\
& \quad \zeta\left(\frac{3}{2}, 1,1,1\right) \\
& \quad=18.11470015628181060307338708154110242297626623122066064185 \ldots,
\end{aligned}
$$

each obtained, via the aforementioned asymmetrical series approach with $L_{1}=$ $2 L_{2}, 20 L_{2}$, and $50 L_{2}$ respectively, in a few minutes on a typical workstation.

\section{ACKNOWLEDGMENTS}

The author is grateful to V. Adamchik, D. Bailey, D. Bradley, J. Buhler, J. Borwein, and D. Broadhurst for their theoretical and computational ideas pertaining to this work.

\section{REFERENCES}

1. D. Bailey, private communication, 1994.

2. D. Bailey, J. Borwein, and R. Crandall, "On the Khintchine Constant," Math. Comp., 66 (1997), 417-431. MR 97c:11119

3. D. Bailey, J. Borwein and R. Girgensohn, "Experimental evaluation of Euler sums," Experimental Mathematics 3, (1994), 17-30. MR 96e:11168

4. D. Borwein, J. Borwein and R. Girgensohn, "Explicit evaluation of Euler sums," Proc. Edinburgh Math. Soc. 38, (1995), 277-294. MR 96f:11106

5. J. M. Borwein and P. B. Borwein, Pi and the AGM, John Wiley \& Sons, 1987. MR 89a:11134

6. J. Borwein and D. Bradley, "Empirically Determined Apery-like Formulae for $\zeta(4 n+3)$," manuscript, 1996.

7. J. M. Borwein, D. M. Bradley and D. J. Broadhurst, "Evaluations of $k$-fold Euler/Zagier sums: a compendium of results for arbitrary $k$," manuscript, 1996.

8. J. M. Borwein and R. Girgensohn, "Evaluation of Triple Euler Sums," manuscript, 1995.

9. D. J. Broadhurst, R. Delbourgo and D. Kreimer, Phys. Lett. B366 (1996), 421.

10. R. E. Crandall, Topics in Advanced Scientific Computation, TELOS/Springer-Verlag, New York, 1996. MR 97g:65005

11. R. E. Crandall and J. P. Buhler, "On the Evaluation of Euler Sums," Experimental Mathematics 3, (1994), 275-285. MR 96e: 11113

12. E. A. Karatsuba, "Fast Calculation of the Riemann Zeta Function $\zeta(s)$ for Integer Values of the Argument s," Probs. Information Trans. 31 (1995), 353-355. MR 96k:11155

13. C. Markett, "Triple Sums and the Riemann Zeta Function," J. Number Theory, 48 (1994), 113-132. MR 95f: 11067

14. D. Zagier, "Values of zeta functions and their applications," preprint, Max-Planck Institut, 1994.

Center for Advanced Computation, Reed College, Portland, Oregon 97202

E-mail address: crandall@reed.edu 